\title{
EXPRESSION OF MATRIX METALLOPROTEINASE 9 IN PATIENTS WITH ORAL LICHEN PLANUS
}

\author{
Vladimíra Paulusovál, Jan Laco², Ivo Dř́zhal', Radovan Slezák ${ }^{1}$
}

\begin{abstract}
Charles University in Prague, Faculty of Medicine and University Hospital in Hradec Králové, Czech Republic: Department
\end{abstract} of Dentistry'; Fingerland Department of Pathology ${ }^{2}$

Summary: Introduction: Oral Lichen planus (OLP) is chronic inflammatory oral mucosal disease of unknown etiology. Basement membrane damage and T-cell migration in OLP may be mediated by matrix metalloproteinases (MMPs). We examined the expression of matrix metalloproteinase 9 to support this hypothesis. Materials and methods: The study population consisted of 71 patients with OLP and 10 control patients with oral fibromas. Indirect immunohistochemistry was used for detection of MMP 9 expression (polyclonal rabbit anti-human MMP antibody). Results: In all cases of OLP, the MMP-9 expression was seen mainly in the area of lymphocytic inflammatory infiltrate in the lamina propria including lymphocytes within the overlying epithelium. In addition, it was observed in the epithelial keratinocytes, particularly in the stratum basale and stratum spinosum with occasional positivity in the superficial layer. Fibroblasts and endothelium of small vessels in the lamina propria showed MMP9 expression as well. In all cases of oral mucosal fibromas, the MMP-9 expression was seen only in fibroblasts and in endothelium of small vessels with occasional positivity within the overlying epithelium. It remains unclear, whether MMP-9 is directly connected to OLP pathogenesis.

Key words: Oral lichen planus; Matrix metalloproteinase 9; Lymphocytic inflammatory infiltrate

\section{Introduction}

Oral lichen planus is a chronic inflammatory disorder that affects the oral mucous membrane. Recently, the classification of OLP tends to be simplified into three major clinical forms (reticular/hyperkeratotic, erythematous/erosive and ulcerative), which could alternate and overlap as the disease progresses (1-3). The prevalence of OLP in the general population is considered to be $1-2 \%(4-7)$. The disease usually manifests at the age of 50-70 years, and is very rare in children (8). Women are more often affected than men (2:1.5) $(9,10)$. Lesions are most often found on the buccal mucosa $(90 \%)$, tongue $(30 \%)$ and gingiva $(13 \%)$. Occasionally they can be also found on the lips and palate $(5,11)$. The appearance of lesions may change during the course of the disease (5). Histological picture is characterized by presence of the subepithelial band-like lymphocytic infitrate and epithelilal basal cell destruction with the formation of apoptotic bodies. The histology and immunohistology strongly support T-cell mediated autoimmune pathogenesis Basement membrane (BM) changes are also common in OLP (13). Hence, keratinocyte apoptosis may be secondary due to BM disruption in OLP lesions. Apoptotic keratinocytes lose the ability to secrete BM structural proteins and are unable to repair damaged extracellular matrix. Both immune antigen-specific as well as general non-specific mechanisms may be involved in the pathogenesis of OLP. Antigen-specific mechanisms in OLP include antigen presentation by basal keratinocytes and keratinocyte killing by CD8+ cytotoxic T-cells. Non-specific mechanisms include for example mast cell degranulation and matrix metalloproteinase (MMP) activation in OLP lesions $(12,13)$.

Matrix metalloproteinases (MMPs) are a zinc-dependent endopeptidases that degrade extracellular matrix and basement membrane components. This group of enzymes is classified as collagenases, gelatinases, stromelysins, membrane-type MMPs and other MMPs. MMPs are involved in physiological processes of tissue remodelling and wound healing and play important role in immune functions (14). MMP-9 is a gelatinase that plays an important role in tissue remodelling in normal and pathological inflammatory processes. It is product of macrophages and a component of cytoplasmatic granules of neutrophils. And is also secreted by stromal cells upon stimulation by inflammatory cytokines (15). The main function of MMP-9 is regulation of cell matrix composition. MMP-9 cleaves denatured collagen and type 4 collagen, which is the major component of the basement membrane. Expression and secretions of MMP-9 by activated lymphocytes and monocytes is tightly regulated by inflammatory cytokines (16). Metalloproteinases also play a role in pathological processes including inflammation, arthritis, cardiovascular diseases, pulmonary diseases and cancer (17). Since the basement membrane disruption is one of the major characteristics of OLP, we wanted to focus on expression of MMP-9 in the OLP lesions. 


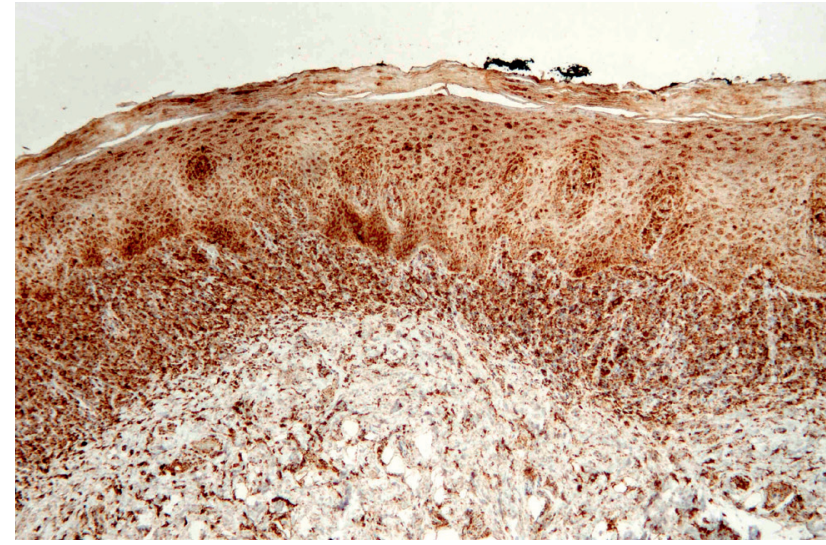

Fig. 1: Expression of MMP9 in OLP in the keratinocytes of stratum basale and stratum spinosum and in the lymphocytes beneath the squamous epithelium (original magnification $100 \times$ )

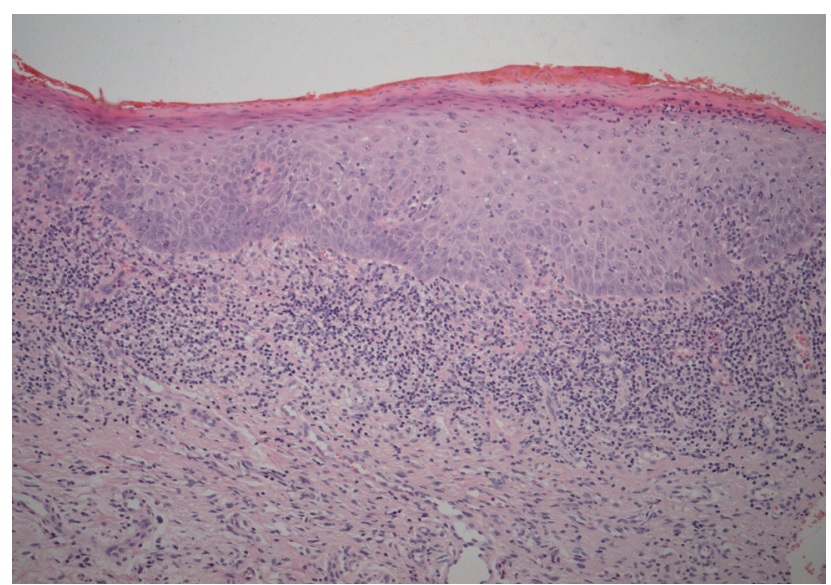

Fig. 3: Microscopic features of lichen planus: mild acanthosis and keratosis of squamous epithelium with band-like lymphocytic infiltrate beneath. The presence of lymphocytes within epithelium is characteristic (hematoxylin-eosin, original magnification $100 \times$ )

\section{Materials and Methods}

A total of 71 tissue samples of oral lichen planus (OLP) were retrieved from the archive files of the Fingerland Department of Pathology, University Hospital, Hradec Kralove, Czech Republic, from the patients who had undergone excision of this lesion at the Department of Dentistry during the years 2002-2009. The specimens were immediately fixed in $10 \%$ formaldehyde, routinely processed, embedded in paraffin and stained with hematoxylin-eosin. All samples were reviewed to confirm the diagnosis of OLP. Ten cases of mucosal fibromas were examined for comparison. The fibroma was chosen because of its non-inflammatory origin. Indirect immunohistochemistry using polyclonal rabbit anti-human MMP9 antibody (code A0150, dilution 1 : 50, Dako (Glos-

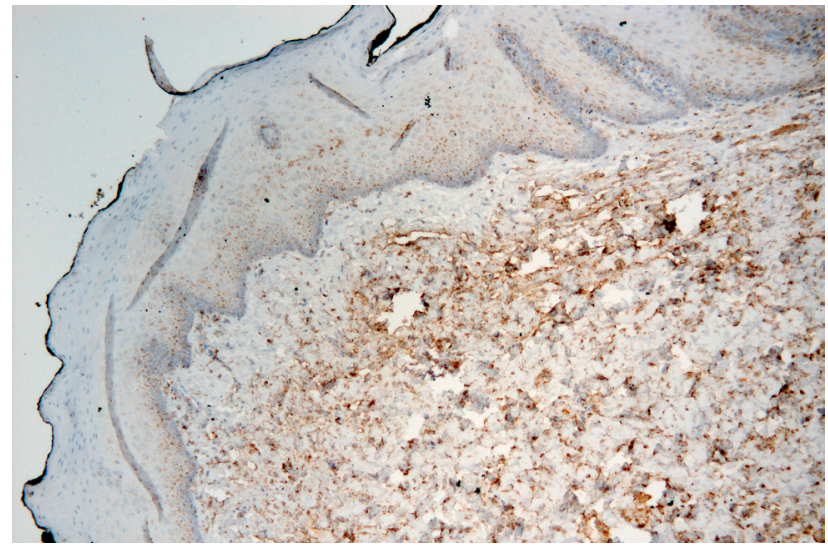

Fig. 2: Expression of MMP9 in oral fibroma in the keratinocytes of stratum basale and stratum spinosum (original magnification $100 \times$ )

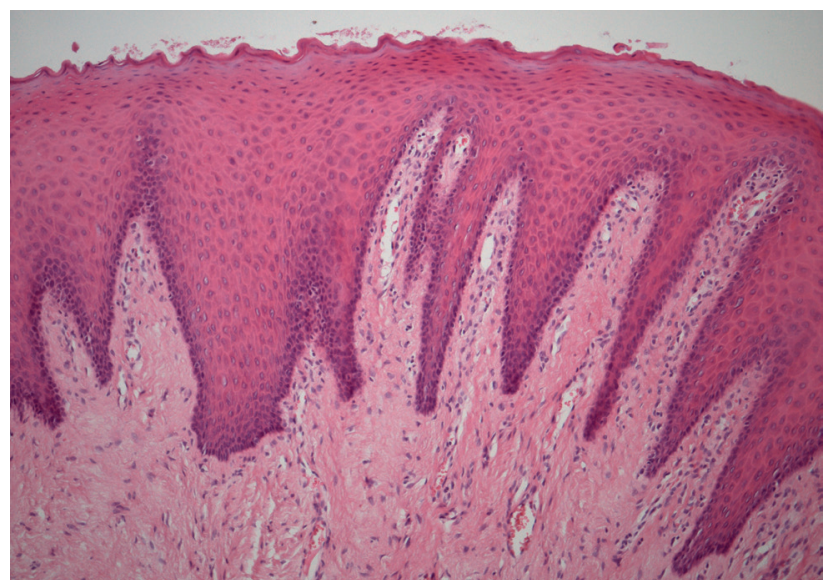

Fig. 4: Mucosal fibroma is composed of fibrous tissue which is covered by acanthotic squamous epithelium (hematoxylin-eosin, original magnification $100 \times$ )

trup, Denmark)) was performed for detection of MMP9 expression. Five- $\mu \mathrm{m}$ sections were cut and mounted on silanized slides. Following blocking of endogenous peroxidase with 3\% hydrogene peroxide in methanol (5 minutes), the sections were incubated with Protein Block Serum (Dako) for 5 minutes. Then, the sections were incubated with diluted primary antibody for 15 minutes. CSA II Rabbit Link, Amplification Reagent and Antifluorescein-HRP (Dako) were incubated for 15 minutes each. During application of the Amplification Reagent, the slides were protected from light. The reaction was visualized using diaminobenzidine. Finally, the sections were counterstained with hematoxylin, causing blue discoloration of cell nuclei). Localization and density of staining were evaluated by light microscopy; the brown staining of cell cytoplasm was considered as positive. 
Specimens of appendix incubated with primary antibody were used as positive controls. Specimens treated with Dako Negative Control, Rabbit Immunoglobulin Fraction instead od primary antibody were used as negative controls.

\section{Results}

In all cases of OLP, the MMP9 expression was seen mainly in the lymphocytic inflammatory infiltrate in the lamina propria including lymphocytes within the overlying epithelium. In addition, it was observed in the epithelial keratinocytes, particularly in the stratum basale and stratum spinosum with occasional positivity in the superficial layer. Fibroblasts and endothelium of small vessels in the lamina propria showed MMP9 expression as well (Fig. 1). In all cases of mucosal fibromas, the MMP9 expression was seen in fibroblasts and in endothelium of small vessels with only occasional positivity within the overlying epithelium (Fig. 2). Hematoxylin eosin photographs of the OLP and fibroma are given for comparison (Fig. 3, 4).

\section{Discussion}

Emerging number of recent studies deal with MMPs as one of the enzyme system in tissue breakdown during pathogenesis of OLP (18-21). Breaking of BM probably leads to apoptosis of keratinocytes, which is typical in OLP and this breakdown is connected to activity of MMPs. Previous studies (18) have for the first time reported possible connection of MMP-2 and OLP. Also other data suggest that increased MMP expression can be seen in squamous cell carcinoma and OLP with OLP having lower expression than carcinoma but higher than normal (19). These results point out two things. MMPs can not only play a role in the pathogenesis of the disease but also promote further invasive behaviour further in the course of the disease and thus cause a transformation of squamous cell carcinoma from OLP. Zhou (20) reported increased expression of MMP-9 in inflammatory infiltrates caused probably by increased secretion of the enzyme from T-cells inside the infiltrate. This situation again leads to breakdown of BM and enhanced keratinocyte apoptosis. In the Gunduz study (21), it was however shown that increased expression of MMP-9 exists also in patients with chronic dermatitis but no subsequent breakdown of basement membrane was shown. This fact can diminish the importance of MMP-9 as a direct mode of action in OLP pathogenesis. Our study group shows distribution of MMP-9 in the lymphocytic infiltrate, the distribution of the MMP-9 in other parts of the specimens is the same compared to oral fibromas. This might represent physiological background of MMP-9 distribution. It remains unclear whether this enzyme truly acts as one of the pathogenetic mechanisms. Antoher issue addressed by recent publication by Chen et al. (22) is the malignant potential of OLP. MMP expression was significantly higher in oral squamous cell carcinoma and atrophic OLP than in nonatrophic OLP and healthy tissue. It is still not generally accepted, that OLP bears the malignant potential and these results might bring more understanding in malignant transformation of OLP. On the other hand, Mazzarella et al. (23) reported higher levels of MMP-9 in reticular OLP than in erosive OLP. Erosive form of OLP is however believed to be more aggressive variant of the disease. These results must be carefully interpreted. There are also several problems connected to the methodology of the research in the field. Almost all studies use different methods of evaluating MMPs. There are quantitative protein expression studies which usually confirmed increased MMP-9 expression in OLP patients. On the other hand, there are immunohistochemistry studies which try to describe the distribution of the enzymes. Both approaches usually confirm increased occurence of MMPs in OLP patients. However, to strictly decide whether MMP-9 is the factor or not is now impossible to say. It could probably be the cooperation of several specific and non-specific mechanisms that cause OLP development, including for example some metabolic pathways (24). MMP-9 is one of the common inflammatory enzymes that not surprisingly is present in OLP samples but its specific role is still discutable. More studies are needed to further describe pathogenetic pathways of OLP.

\section{Acknowledgements}

This work was supported by research project MZO 00179906. The authors report no conflicts of interest.

\section{References}

1. Piboonniyom SO, Treister N, Pitiphat W, Woo SB. Scoring system for monitoring oral lichenoid lesions: a preliminary study. Oral Surg Oral Med Oral Pathol OralRadiol Endod 2005; 99: 696-703.

2. Wright J. Oral lichen planus. Br Dent J 2004; 197: 224-5

3. Karatsaidis A, Schreurs O, Helgeland K, Axell T, Schenck K. Erythematous and reticular forms of oral lichen planus and oral lichenoid reactions differ in pathological features related to disease activity. J Oral Pathol Med 2003; 32(5): 275-81.

4. Eisen D. Evaluating and treating patients with oral lichen planus. Dermatol Ther 2002; 15: 206-17.

5. Dissemond J. Oral lichen planus: an overview. J Dermatol Treat 2004; 15: 136-40.

6. Sugerman PB, Savage NW, Zhou XJ, Walsh L, Bigby M. Oral lichen planus. Clin Dermatol 2000; 18: 533-9.

7. Axell T, Rundquist L. Oral lichen planus-a demographic study. Commun Dent Oral Epidemiol 1987; 15: 52-6.

8. Silverman S Jr, Gorsky M, Lozada-Nur F. A prospective follow-up study of 570 patients with oral lichen planus: persistence, remission, and malignant association. Oral Surg Oral Med Oral Pathol 1985; 60: 30-4.

9. Eisen D. The clinical features, malignant potential, and systemic associations of oral lichen planus: a study of 723 patients. J Am Acad Dermatol. 2002; 46: 207-14.

10. Mollaoglu N. Oral lichen planus: a review. Br J Oral Maxillofac Surg. 2000; 38 : 370-7.

11. Scully C, Beyli M, Ferreiro MC et al. Update on oral lichen planus: Etiopathogenesis and management. Crit Rev Oral Biol Med 1998; 9: 86-122.

12. Sugerman PB, Savage NW, Walsh LJ et al. The pathogenesis of oral lichen planus Crit Rev Oral Biol Med 2002; 13: 350-65.

13. Roopashree MR, Gondhalekar RV, Shashikanth MC, et al. Pathogenesis of oral lichen planus-a review. J Oral Pathol Med. 2010; 39: 729-34.

14. Sorsa T, Tjäderhane L, Salo T. Matrix metalloproteinases (MMPs) in oral diseases. Oral diseases 2004; 10: 311-318.

15. St-Pierre Y, Themsche CV, Esteve P-O. Emerging feature in the regulation of MMP-9 gene expression for the development of novel molecular targets and therapeutic strategies. Curr Drug Targets Inflamm Allergy 2003; 2: 206-15.

16. Ram M, Sherer Y, Shoenfeld Y. Matrix metalloproteinase-9 and autoimune diseases. J Clin Immunol 2006; 26: 299-307. 
17. Chakraborti S, Mandal M, Das S, et al. Regulation of matrix metalloproteinases: an overview. Mol Cell Biochem. 2003; 253: 269-85.

18. Giannelli G, Brasssard J, Foti C, et al. Altered expression of basement membrane proteins and their integrin receptors in lichen planus: possible pathogenetic role of gelatinases A and B. Lab Invest 1996, 74: 1091-104.

19. Sutinen M, Kainulainen T, Hurskainen T, et al. Expression of matrix metalloproteinases (MMP-1 and -2) and their inhibitors (TIMP-1, -2 and -3 ) in oral lichen planus, dysplasia, squamous cell carcinoma and lymph node metastasis. $\mathrm{Br}$ Cancer 1998; 77: 2239-45.

20. Zhou XJ, Sugerman PB, Savage NW, et al. Matrix metalloproteinases and their inhibitors in oral lichen planus. J Cutan Pathol. 2001; 28: 72-82.
21. Gunduz K, Demireli P, Inanir I, et al. Expression of matrix metalloproteinases (MMP-2, MMP-3, and MMP-9) and fibronectin in lichen planus. J Cutan Pathol 2006; 33: 545-50.

22. Chen Y, Zhang W, Geng N, et al. MMPs, TIMP-2, and TGF-betal in the cancerization of oral lichen planus. Head Neck. 2008; 30: 1237-45.

23. Mazzarella N, Femiano F, Gombos F, et al. Matrix metalloproteinase gene expression in oral lichen planus: erosive vs. reticular forms. J Eur Acad Dermatol Venereol. 2006; 20: 953-7.

24. Paulusová V, Rösch C, Dřízhal I, et al. Cytochrome P450 2D6 polymorphism and drug utilization in patients with oral lichen planus. Acta Odontol Scand 2010; 68: $193-8$.

Received: 12/09/2011

Accepted in revised form: 07/11/2011

\section{Corresponding author:}

Vladimíra Paulusová, M.D., University Hospital, Sokolská 581, 50005 Hradec Králové, Czech Republic, Department of Dentistry; e-mail: vladimira.paulusova@fnhk.cz 